\title{
Indice Ponderado de Importancia Turística: Herramienta para Decisiones Estratégicas y Políticas
}

\author{
Alfredo Ascanio ${ }^{1}$
}

RESUMEM:Elartículodiscuteuna herramienta de análisis relativa a la importancia del turismo cuando se hacen comparaciones entre paises competitivos. El modelo que se desarrolla utiliza un índice ponderado, que se basa en comparar un grupo de variables o indicadores contra una norna de equilibrio. Los resultados del modelo ayudan a tomar decisiones estratégicas en cuanto a loselementosque sedeben potenciar para elevar el índice, establecerpoliticas y lograr de este modo una mayor competitividad turística en relación a otros países de una región deternninada.

PALAVRAS CLAVES: Competitividad turistica; indice ponderado; América; Venezuela.

ABSTRACT: This article shows an analysis of tourism importance when comparison was made between competitive countries. The model developed used compound index of variables and indicators. The model results helps to take strategic decisions to improve variables to establish policies, in order to be more competitive in a specific region.

KEY WORDS: Competitive tourism; compound index; America; Venezuela.

1. Economista e pós-graduado em Havard. Professor da Universidade Simón Bolivar, Caracas, Venezuela. Ex-assessor de Organismos Intemacionais de Turismo.

End. para corresp.: Urb. Alto Proda - Calle 10 y Av. 12 - Qto. Los Helechos - Caracas 1080 - Venezuela. 


\section{Introducción}

Las instituciones encargadas de administrar los recursos turísticos de una manera efectiva, seenfrentan a la conquista de mercadosen un mundo competitivo. Una manera de comparar la posición relativa del turismo en un pais determinado, con un conjunto de países, que de alguna manera son competidores entre sí, no es estableciendo una orden de jerarquía turística solamente basado en el mayor o menor número de turistas captados y en los ingresos que ese turismo produce, sino analizando un conjunto coherente de variables que permitan formar un indice ponderado de la importancia del turismo, para poder hacer una comparación entre países competidores; y discutir, luego, una estrategia integral y sus políticas, a fin de lograr una mejor posición a mediano y largo plazo.

¿Cuáles son las variables más importantes que deben ser consideradas para hacer esta comparación? ¿Cuál es el peso que debe recibir cada variable? y, ¿Cuál es la norma de equilibrio que permitiría conocer si cada variable se aleja o se acerca de dicha norma? En seguida se discuten estos asuntos y se explica, paso a paso, la construcción de un índice que permita hacer una comparación entre países competitivos, a fin de mejorar la importancia del turismo.

\section{Definiciones y Aspectos Conceptuales}

Es necesario definir algunos de los términos utilizados en este artículo. Un indice es un instrumento de medición que consta de varios itens, que llamaremos indicadores, y que perniten medir un concepto, como la importancia del turismo, por ejemplo, desde un punto de vista empírico (Mora y Araujo, 1971: 19). La solución mas común consiste en algun tipo de combinación sumatoria de los indícadores para formar con éllos un índice.

¿Qué datos se podrian selecionar para laconstrucción delíndice? Enestecaso los indicadores deben medir la importancia del turismo y por ello existe la posibilidad de elegir entre un número teóricamente vastíssimo de indicadores posibles²; pero los indicadores que vamos a nombrar en seguida, forman parte de un marco teórico o referencial que considera al turismo desde dos ángulos importantes como son: el aspecto económico y el ambiental. En efecto, casi todos los países se interesan por el turismo, porque produce riqueza y porque esa riqueza producida deberia obtenerse en armonía con el medio.Podemos entonces seleccionar algunos indicadores que siendo teóricamente dependientes, se adecuen bien al marco económico-ambiental señalado, como serían por ejemplo: el ingreso por turista y viaje, el ingreso turístico per capita, la importancia del gasto turismoreceptivo en relación a las exportaciones,

2. En el informe estadístico del Caribbean Tourism Organization (1995), se utilizan además de los indicadores que se reseñan en este artículo, dos adicionales que tienen relación con la hotelería, o sea: el ingeso gubernamental debido al impuesto hotelero y el número de cuartos por 1.000 habitantes. la incidencia del gasto turístico emisivo en relación a las importaciones, así como la importancia del turismo en relación a la economía de los servicios; y por supuesto el número de turistas por mil habitantes y por $\mathrm{km}^{2}$ de superficie.

Los indicadores que permiten construír índices o escalas turísticas, son entonces propiedades manifiestas, u observables, que se suponen sondatosempíricos proporcionados por las estadísticas y que deben estar referidos a un concepto, que a su vez tiene que ver con un marco referencial relevante. En general, los valores de un índice son más adecuados para realizar inferencias, que los valores de un indicador aislado (Mora y Araujo, 1971: 11). El índice que queremos estudiar, es una sumatoria pesada o ponderada de indicadores, según su mayor o menor importancia, a fin de obtener un dato acumulativo que permita jerarquizar a los países, según su importancia turística económica-ambiental.

Según el procedimiento, los indicadores seleccionados se combinan de alguna manera para construír el índice. La construcción de los instrumentos de medición es un momento crucial, pues debe existir relación entre los indicadores seleccionados, el concepto que deseamos medir y su marco teórico. En este caso es necesario, entonces, que tengamos bien claro lo que significa para un país ser "importante turísticamente". Por ejemplo, en este caso se parte de un criterio de importancia, como ya fue señalado, que se relaciona con cuatro aspectos básicos, próximos a la experiencia general oincluso a valores formuladosen la literatura relativa al turismo, como son:

- los países se interesan por captar un turista de alto poder adquisitivo y que pueda permaneceren el sitio durante una larga estadía; o puedaser reemplazado por otro turista, en un breve espacio de tiempo:

- los países se interesan también, porque el volumen de captación de ingresos turísticos sea elevado, en relación al conjunto de ingresos de la economía de los servicios; eincluso, encomparación conlosingresosque producen las exportaciones no tradicionales;

- los países, a fin de mantener una Balanza de Viajeequilibrada, trataríande obtener por lo menos la misma cantidad de ingresos por concepto de turismo receptivo, que los egresos que tendrían que erogar debido al turismo emisivo;

- los países desearían un turismo de baja densidad, en cuanto a la ocupación de un espacio geográfico determinado; y que no produzca un desplazamiento de la población anfitriona, en cuanto al uso de los servicios, a los cualestienen derecho por ser residentes.

Las anteriores hipotesis auxiliares deben funcionar, si suponemos que la hipótesis sustantiva es que la relación entre ese conjunto de indicadores nos debe acerca bastante bien a la idea de la importancia turística. Un índice ponderado de importancia turística, que no guarde relación alguna con el pesoque un paístieneen el mercado turístico, sería difícilmente aceptable.

En la medida en que el concepto que queremos medir sea mas complejo, necesitaremos un mayor número de indicadores, ya que el concepto no se podría 
medir con una sola variable, como se viene haciendo tradicionalmente en turismo, cuando se nos señala que un determinado pais es muy importante turisticamente porque alli llegan millones de turistas; y esa economía se beneficia también de millones de dólares al año, indicadores individuales bastante circunstanciales, pues ello depende de un contexto muy cambiante y competitivo; aunque es posible que esa relación entre la importancia turística y la llegada de alıs volumenes de turistas y de ingresos, no sea probalilística sino determinista, como a menudo sucede con los paises altamente desarrollados, donde casi permanentemente se arrojan cifras importantes acerca de su economía turistica, ofertan amplios espacios ambientales y sus servicios pueden satisfacer no sólo a los residentes, sino a una población temporal, que aunque les eleva la capacidad de carga, no producen aglomeraciones y deficits perjudiciales; entonces, en estos países si sería válido utilizar esos indicadores de una manera aislada, pero este no es el caso de los países en vias de desarrollo, donde la data turística puede ser fluctuante o en países muy pequeños, como las islas del Caribe, donde la población residente no es elevada y el espacio geográlico cs bastante reducido.

\section{Selección de Indicadores}

La selección de los indicadores es una de las fases para las cuales menos técnicas precisas o sistemáticas. Habitualmente la opinión de los expertos, ayuda en este proceso de selección, asi como en la asignación de los pesos o ponderaciones a cada variable. La pregunta que nos debemos hacer es: si los indicadores seleccionados son lo baslante representativos como para formar el indice que se debe relacionar con el concepto en estudio. Por lo tanto, la inclusión de un indicador es una decisión de tipo teórico, que debe luego ser confirmada o rechazada empíricamente (Korn, 1969: 11).

Si los indicadores, que sirven para la construcción del indice, estan expresados en promedios y otros en porcentajes, es necesario "relativitar" estas medidas con respecto a un valor de referencia, que en muesiro caso será una norma de equilibrio, la cual puedesurgir de un valor promediobien sea nacional o regional (Briones, 1982: 169).

\section{Selección de la Norma de Equilibrio}

En los párrafos anteriores debc liaber quedado claro que la diferencia entre un índice y $u n$ indicador es de grado, pucs cl indice es un complejo de indicadores de diferentes valores y constituye, por lo tanto, el indicador total de una variable compleja.

Pero como algunos indicadores se pueden expresar en promedios y otros en porcentajes, sólo serian comparables si los relacionamos con un indicador global que provenga de un conjunto amplio de sujetos, a los fines de que sea una representación casi balanceada o equilibrada de muchos valores diferentes. En cste caso, estariamos comparando cada indicador con un valor más colectivo, que posea una propiedad globalizada; es decir, que puede ser definido por alguna propiedad ajena a las propiedades de los sujetos que le dieron origen, como sería la propiedad de guardar un equilibrio, ya que no debería situarse en ningún valor extremo.

La norma que nos interesa entonces, en este caso, es un valorglobal parecido al valor de la mediana, que nos estaria indicando que un pais podria tener importancia turistica balanceada, siempre y cuando no caiga en valores extremos que lo configuren o bien como un monoproductor turístico, lo cual implicaría mucho riesgo; o bien, como un país donde el turismo casi no existe o por lo menos es muy incipiente, lo cual tambien no pareciera conveniente en cl contexto actual de sociedades modernas y lúdicas.

\section{Ejemplo sobre la Utilización del Índice}

En seguida aparece la utilización del índice como un elemento para diagnosticar la competitividad turistica de un país. En este caso vamos a utilizar los estadisticos del año 1993 y la posición que ocupaba Venezuela en relación a otros países de la región de las Américas.

Los indicadores utilizados son los siguientes:

- la importancia del turismo desde el punto de vista del ingreso por turista y viaje; ya que es posible que a un país llegue una cuantidad menor de luristas, pero que ello se compensa con la captación de un turismo de elevado gasto diario y alta estancia media;

- el ingresoturístico per capita, ya que en la medida e

netos turísticos esto ticne que guardar una armonúa con la cantidad de poblacion, a los fines de establecer una mejor política redistributiva del ingreso turistico;

- la relación entre los ingresos turísticos y el PIB de los servicios, pues estc indicador sí es equilibrado, demonstraría la armonía entre cl turismo y los otros servicios;

- la relación entre los ingresos del turismo receptivo y las exportaciones no tradicionales, pues debe existir un buen equilibrio entre todos los rubros que exporta un país en su esfuerzo de diversificar su economía;

- la relación cntre los egresos turisticos y las importaciones, de manera tal que la erogación de divisas turisticas hacia el Exterior no este desbalanceada con 1 erogación de divisas por otros motivos; y que responda a un comercio internaci onal turístico recional con los países con los cuales mantenemos intercambios;

- el número de turistas por 1.(0)() habitantes y el númerode turistaspor $\mathrm{km}^{2}$, a los fines de controlar la densidad turística y evitar problemas de aglomeración, déficits de servicios y externalidades negativas.

En seguida aparecen los pesos otorgados a cada indicador y la norma de equilibrio para 1993 (Quadro 1), que sirve de modelo de comparación, ya que un pais 
que logre armonizar todos sus indicadores pesados, con relación a esa norma de equilibrio, obtendría un indice igual a 100; en cambio un país que se coloque por debajo del indice 100 , debería realizarun esfuerzo de marketing y de inversiones, para elevar su índice por lo menos hasta 100; y todo país que arroje un índice mayor de 100 , podría estar acercándose a una situación, donde el turismo comenzaría a ser un sectoreconómicotanpredominante, que ese paíspodria llegara serun monoproductor turístico si el índice se coloca en una cifra parecida a la de la mayoría de las islas del Caribe insular, con índices que van desde 3 veces hasta más de 47 veces la norma de equilibrio iguala 100.

QUADRO 1 - PESO E NORMA DE EQUILIBRIO POR INDICADOR VENEZUELA, 1993

\begin{tabular}{l|c|c}
\hline Indicadores & $\begin{array}{c}\text { Norma de } \\
\text { Equilibrio } \\
\text { (1993) }\end{array}$ \\
\hline Ingreso por turista y viaje & 30 & US $\$ 820$ \\
Ingreso turístico per capita & 20 & US $\$ 266$ \\
Ingreso turístico como \% del PIB de los servicios & 10 & $11 \%$ \\
Egreso turístico como \% de las exportaciones & 10 & $15 \%$ \\
Turismo como \% de las importaciones & 6 & $6 \%$ \\
Ingreso per capita turistico del per capita general & 10 & $5 \%$ \\
Llegadas de turismo por 1.000 habitantes & 8 & 172 turistas \\
Llegadas de turismo por $\mathrm{km}^{2}$ & 6 & 5 turistas \\
\hline
\end{tabular}

Como se puede observar en los datos anteriores, los pesos que se han otorgado a cada indicador es un juicio de valor en lo referente a la importancia económica de cada elemento; y a la cautcla que es necesario tener, en lo referente a la erogación de divisas turisticas y a la densidad turística, como se señalo en párrafos anteriores.

\section{La Manera de Calcular el Îndice}

El índice no es más que el resultado de realizar una sumatoria de todos los indicadores de un país determinado, comparado cada indicador con la norma; y dándole, luego, el peso correspondiente. Esdecir, primero se calculaqué porcentajes representan los diversos valores del país analizado, en relación con la norma de equilibrio establecida (en otras palabras, el valor de equilibrio se hace igual a 100); y luego, se multiplica por el peso correspondiente, como aparece en seguida para el caso venezolano y para los datos de 1993 (Quadro 2).
QUADRO 2-CONSTRUCCIÓN DEUN INDICE DE LA IMPORTANCIA PONDERADA DEL TURISMO PARA VENEZUELA - 1993

\begin{tabular}{|c|c|c|c|c|}
\hline Indicadores & $\begin{array}{c}\text { Valores } \\
\text { observados } \\
\text { (a) }\end{array}$ & $\begin{array}{c}\text { Valor de la } \\
\text { norma (b) } \\
\text { (b) }\end{array}$ & $\begin{array}{r}\% \text { de (a) } \\
\text { entre (b) }\end{array}$ & Peso \\
\hline $\begin{array}{l}\text { a) Ingreso por } \\
\text { turismo y viaje }\end{array}$ & 1.399 & 820 & 1,71 & 30 \\
\hline $\begin{array}{l}\text { b) Ingreso turistico } \\
\text { per capita }\end{array}$ & 26 & 266 & 0,10 & 20 \\
\hline $\begin{array}{l}\text { c) Turismo como } \% \text { del } \\
\text { PIB de los servicios }\end{array}$ & 1,71 & 11 & 0,10 & 10 \\
\hline $\begin{array}{l}\text { d) Ingreso turistico } \\
\text { como \% de las } \\
\text { exportaciones }\end{array}$ & 3,95 & 15 & 0,26 & 10 \\
\hline $\begin{array}{l}\text { e) Egreso Turistico } \\
\text { como \% de las } \\
\text { importaciones }\end{array}$ & 11,04 & 6 & 1,84 & 6 \\
\hline $\begin{array}{l}\text { f) Ingreso per capita } \\
\text { turistico del per capita } \\
\text { general }\end{array}$ & 0,91 & 5 & 0,18 & 10 \\
\hline $\begin{array}{l}\text { g) Llegadas de turistas } \\
\text { por } 1.000 \text { habitantes }\end{array}$ & 19 & 172 & 0,11 & 8 \\
\hline $\begin{array}{l}\text { h) Llegadas de turistas } \\
\text { por } \mathrm{km}^{2}\end{array}$ & 0,44 & 5 & 0,09 & 6 \\
\hline
\end{tabular}

Con las relaciones entre (a) y (b) anteriores y los pesos o ponderaciones, podemoscalcular el IPIT (ÍndicePonderadode Importancia Turística para Venezuela) de esta manera:

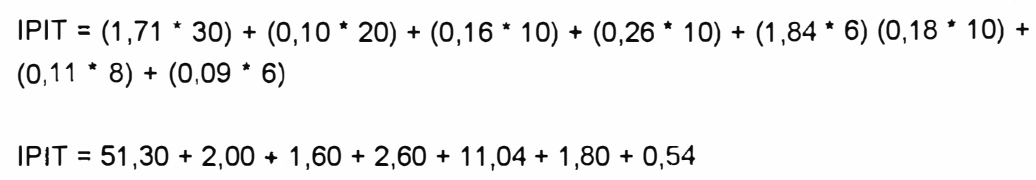

IPIT $=71,76($ digamos $72 \%)$ 
EI IPIT para Venezuela nos indica que el pais hasta 1993 habia logrado un $72 \%$ en relación a la norma de cquilibrio del $100 \%$, por lo que aún le faltaba al país hacer un esfuerzo adicional del $28 \%$ en estratégias y políticas cspecíficas; lo cual podría lograrse con un mejor control de su Balanza de Pago de Viajes, especialmente con países como Estados Unidos de Norteamerica, Italia, Aruba, Curazao, España, Portugal, Inglaterra, Francia y'Brasil; así como elevar la llegada de turistas por 1.000 habitantes y' también cl ingreso lurístico como \% de los servicios, estableciendo una meta a mediano plazo, para poder alcanzaral año por lomenos 1 millón deturistas de alto poderde gasto y de una estancia media relativamente clevada, cspecialmente de aquellos países emisores con los cuales tenemos Balanza de Pago deficitaria.

Si el índice de Venezuela se compara con el índice de otros países de las Américas, podemos encontrar los siguientes hallazgos (Quadro 3)

QUADRO 3 - INDICE PONDERADO DE IMPORTANCIA TURISTICA (IPIT) DEL TURISMO RECEPTIVO PARA LOS,PRINCIPALES PAISES DE LAS AMÉRICAS - 1993

\begin{tabular}{lcc}
\hline País & Indice & Posición Relativa \\
\hline Aruba & 4.787 & 1 \\
Islas Virgenes (USA) & 2.779 & 2 \\
Bahamas & 2.478 & 3 \\
Guadalupe & 742 & 4 \\
Puerto Rico & 504 & 5 \\
Jamaica & 290 & 6 \\
Republica Dominicana & 237 & 7 \\
Cuba & 127 & 8 \\
Costa Rica & 92 & 9 \\
Uruguay & 88 & 10 \\
USA & 85 & 11 \\
Venezuela & 72 & 12 \\
Canada & 70 & 13 \\
Argentina & 60 & 14 \\
México & 57 & 15 \\
Guatemala & 46 & 16 \\
Chile & 40 & 17 \\
Brasil & 39 & 18 \\
Ecuador & 38 & 19 \\
Colombia & 35 & 20 \\
\hline
\end{tabular}

( ${ }^{a}$ Sin considerar el turismo fronterizo en México
En forma grafica cl IPIT aparece como sigue, en la Figura I.

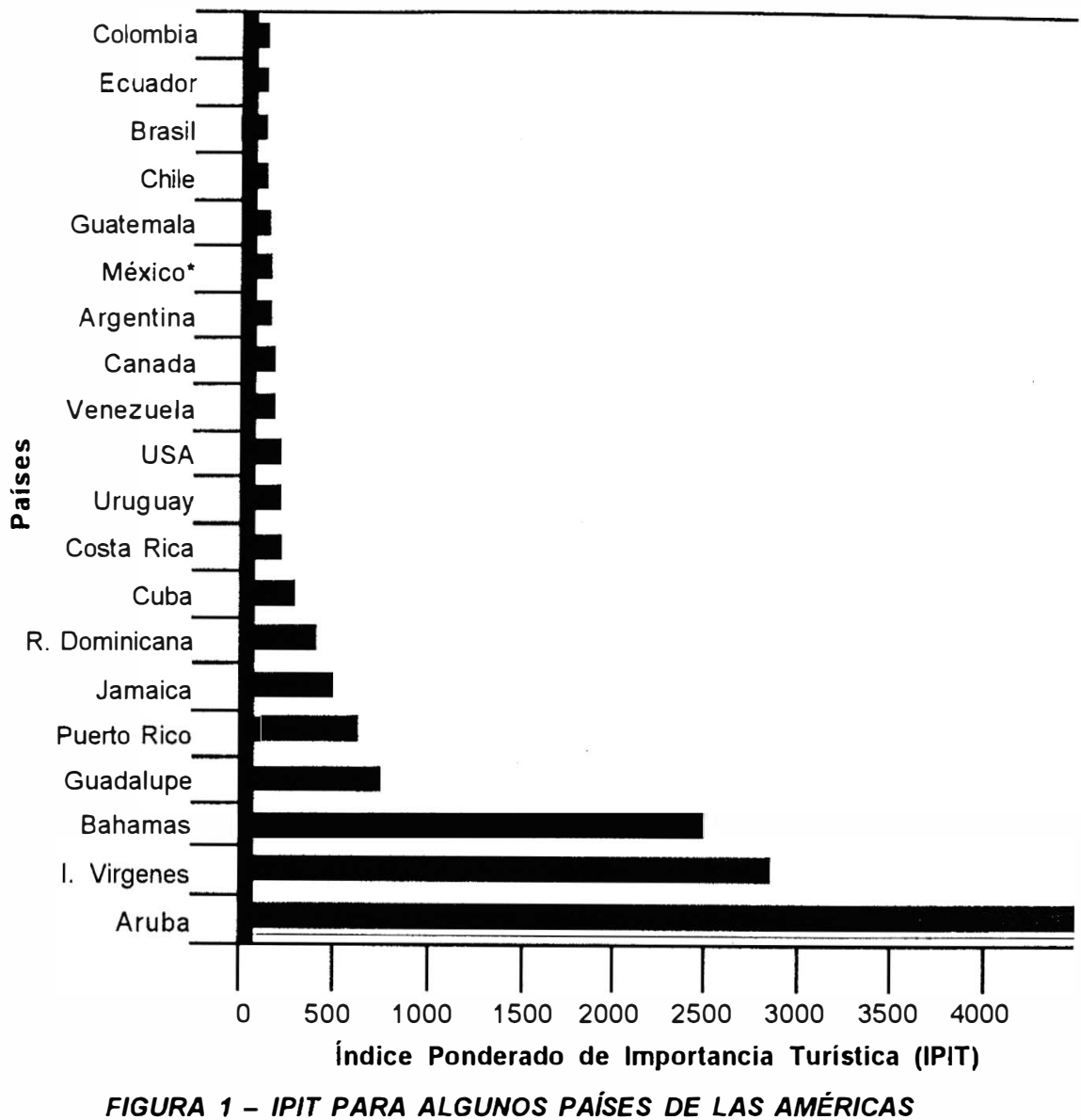

Conclusión

El Índice Ponderado de Importancia Turistica (IPIT) puede transformarse en una herramienta analítica para establecer estratcgias y políticas. Este índice conjuntamente con otros indices, como porejembro cl desarrollado por Wynegar (497-508), de la Oficina de Investigación del United States Travel and Tourism Administration, para la evaluación del potencial del mercado turístico internacional, se convierten en elementos básicos para la toma de decisionesa nivel de las Oficinas Gubernamentales de Turismo. 


\section{Bibliografía}

ASCANIO, Alfredo. 1994. Concentración espacial y rendimiento turístico en bloque de países. Un dilema para la competitividad. Revista Argos, Caracas, n. 20, Nov-Dic.

BRIONES, Ciuillemo. 1982. Métodos y técnicas de imvestigación para las ciencias sociales, México: Editorial Trillas.

C:ARIBBEAN TOURISM OR(.AANIZATION (CTO). 1995. Caribbean tourism statistical report.

KORN, Francis. 1969. La significación del témino variable en sociología. En: Conceptos y variables en la imestigación social. Buenos Aires: Nueva Visión

MORA Y ARAUJO, Manuel. 1971. Introducción a la medición y construcción de indices. Buenos Aires: Nueva Visión.

IVYNEGAR, Done. Estimating the potential of international markets. En: Tran'el, tourism and hospitality research. A handbook for managers and researchers. New York: John IViley de Son.

Recebidlo em $9 / 4 / 97$

Aprovado em 11/8/97 